\title{
Discrepancy-Based and Anticipated Emotions in Behavioral Self-Regulation
}

\author{
Christina M. Brown \\ Saint Louis University
}

\author{
Allen R. McConnell \\ Miami University
}

\begin{abstract}
Discrepancies between one's current and desired states evoke negative emotions, which presumably guide self-regulation. In the current work we evaluated the function of discrepancy-based emotions in behavioral self-regulation. Contrary to classic theories of self-regulation, discrepancy-based emotions did not predict the degree to which people engaged in self-regulatory behavior. Instead, expectations about how future self-discrepancies would make one feel (i.e., anticipated emotions) predicted self-regulation. However, anticipated emotions were influenced by previous discrepancy-based emotional experiences, suggesting that the latter do not directly motivate self-regulation but rather guide expectations. These findings are consistent with the perspective that emotions do not necessarily direct immediate behavior, but rather have an indirect effect by guiding expectations, which in turn predict goal-directed action.
\end{abstract}

Keywords: self-regulation, emotions, self-discrepancies, expectations, affective forecasting

Emotions have long been assumed to guide behavior through their motivational properties. For example, emotions are thought to evoke behavioral impulses, with each emotion priming "readiness" for certain actions (Frijda, 2000). Moreover, some theorists believe the ultimate function of emotions is to activate and direct goalrelated behaviors (Cosmides \& Tooby, 2000). Although individuals who have impaired emotional experiences reveal the necessity of emotions for optimal functioning (Damasio, 1994), there is far more theory than evidence suggesting that emotions directly guide goal-directed behavior.

In the present research we examined the role of emotions in goal-directed behavior, also known as self-regulation. According to classic theories of self-regulation (e.g., Carver \& Scheier, 1999; Duval \& Wicklund, 1972; Higgins, 1987), people self-regulate when they try to reduce a discrepancy between their current state and a desired state. Discrepancies between current and desired states elicit negative emotions, which are believed to guide goaldirected behavior. A common view is that negative emotions directly motivate self-regulation because people want to eliminate the negative feelings by removing their source (i.e., the discrepancy; Duval \& Wicklund, 1972). Although there is wide agreement that emotions play a key role in self-regulation, there is little empirical evidence of a relation between one's current emotional experiences and self-regulatory behavior. For example, research on cognitive dissonance is consistent with the proposal that negative emotions motivate behavioral change (e.g., Cooper \& Fazio,

This article was published Online First April 25, 2011.

Christina M. Brown, Department of Psychology, Saint Louis University; Allen R. McConnell, Department of Psychology, Miami University.

Portions of this work were included in a dissertation by the first author submitted to Miami University in partial fulfillment of the requirements for a doctoral degree.

Correspondence concerning this article should be addressed to Christina M. Brown, Department of Psychology, Saint Louis University, 221 North Grand Boulevard, St. Louis, MO 63109. E-mail: cbrown81@ @lu.edu
1984), but such a relation has not been shown in other domains of goal pursuit.

An alternative to this motivational account is that discrepancybased emotions do not guide immediate self-regulation, but rather stimulate learning and inform future decisions to self-regulate. Recently, Baumeister and colleagues (Baumeister, Vohs, DeWall, \& Zhang, 2007) argued against the classic view that emotions directly cause behavior (e.g., fear makes a person flee). Instead, they view emotion as a feedback system that stimulates learning about the situation that elicited the emotion (i.e., people learn to associate the situation and emotion). This learning enables people to anticipate the emotions they will feel in particular situations, with these anticipated emotions guiding subsequent behavior (that is, actions are based on the emotions people expect to feel). When applied to self-regulation, this learning account of emotion predicts that discrepancy-based emotions function to stimulate learning, with anticipated (but not current discrepancy-based) emotions guiding immediate self-regulation.

We compared these two accounts of the role of emotion in self-regulation. According to a motivational account, the negative emotions people feel following a self-discrepancy should directly predict subsequent self-regulation efforts. In contrast, a learning account predicts that self-regulation will be guided by how people expect to feel after a self-discrepancy, and that discrepancy-based emotions should inform these anticipated emotions. The current study evaluated these two accounts by creating multiple instances in which participants experienced a self-discrepancy and reported their emotions. This allowed us to assess whether previous discrepancy-based emotions predicted subsequent anticipated emotions, as predicted by the learning perspective (i.e., the function of current emotions is to inform expectations of one's future emotions). In addition, we examined whether anticipated emotions became more accurate over time, which would suggest that people actively learn from their previous emotional experiences. This prediction is important given that affective forecasting research shows that people are often inaccurate when predicting how cer- 
tain events will influence their emotions (e.g., Wilson \& Gilbert, 2003). Yet, if the learning account is correct and current emotions stimulate learning by informing subsequent anticipated emotions, then experiencing a self-discrepancy and its corresponding negative emotions should increase accuracy of future predictions about how self-discrepancies will make one feel (i.e., experience should reduce affective forecasting errors). In sum, we tested (1) if emotions following a self-discrepancy predict self-regulation, (2) if how people expect to feel (anticipated emotions) predicts selfregulation, (3) if recent discrepancy-based emotions predict subsequent anticipated emotions, and (4) if experiencing discrepancy-based emotions improves the accuracy of anticipated emotions.

\section{Method}

\section{Overview}

To explore the temporal relations between discrepancy-based emotions, anticipated emotions, and self-regulation, we twice induced a self-discrepancy and measured participants' subsequent current and anticipated emotions. The self-discrepancy was created by giving participants noncontingent failure feedback about their performance on an important test. We then measured selfregulation by giving them the opportunity to improve their ability through practice, with more practice representing greater selfregulation. Next, participants completed another test and received failure feedback a second time. Afterward, they reported their emotions again with the belief that they would take a third test.

\section{Participants}

Participants were 221 undergraduates who received research credit in return for their participation. Fifteen participants were excluded from analyses for the following reasons, which were revealed during debriefing: six admitted to responding randomly (i.e., pressing random buttons), five reported preexisting extreme moods (e.g., one participant reported having just broken up with his girlfriend), and four were suspicious of the failure feedback. Two additional participants had practice effort scores that were outliers (i.e., more than three standard deviations from the mean). After removing these participants, a total of 204 (138 women, 65 men, 1 undisclosed) participants remained.

\section{Measures}

Emotion. Emotions were measured using a modified "state" version of the Basic Emotions Scale (BES; Power, 2006), which contains 20 adjectives (e.g., frustrated, cheerful) that represent five discrete emotions: anger, sadness, self-disgust, fear, and happiness. For each measure of current emotions, participants indicated the extent to which they currently felt each emotion on a scale ranging from 1 (not at all) to 7 (very much). For the measure of anticipated emotions, participants reported how they expected to feel if they failed the upcoming test using the same scale.

Self-regulation. Participants were given the opportunity to practice before the second supposed assessment of their ability. They could practice as much as they wanted, for a maximum of either $15 \mathrm{~min}$ or for 60 problems (whichever came first). The computer program allowed participants to exit the practice task whenever they desired. The amount of time and number of problems practiced were recorded.

\section{Procedure}

Participants completed the experiment on computers in private rooms. They were told their social perception skills would be tested using an emotion recognition task. To make the task important, participants were told,

The ability to identify the emotions and feelings of other people is an essential skill for successful friendships, relationships, and careers. Being able to detect how someone is feeling helps you avoid potential conflict with them and respond to their needs.

In addition, participants were told that they would complete three social perception tests (in actuality, they completed two), that they would receive feedback concerning their performance on each test, and that they would have the opportunity to practice emotion recognition problems before the second and third tests (in fact, they only practiced once because there was no third test).

Each "social perception" test consisted of 10 photos (obtained from the Productive Aging Lab face database; Minear \& Park, 2004) of people posing neutral expressions. Participants were led to believe that each photographed person was experiencing an emotion but had been instructed to mask his or her feelings. Participants were told to select which emotion (out of happiness, sadness, anger, frustration, guilt, anxiety, or pride) they thought the person was experiencing. In actuality, there were no correct answers because each person displayed a neutral expression, making the task ambiguous and increasing the believability of the failure feedback.

Participants began the study by completing an initial measure of their emotions and the first measure of anticipated emotions (i.e., how they expected to feel if they failed the first test), which we will refer to as Baseline emotions. Participants then completed the first social perception test, which was followed by failure feedback. Specifically, the computer reported that they answered three of the 10 problems correctly and read, "This score means your social perception skills are poor." They then completed the second measure of current and anticipated emotions (Posttest 1 emotions). Next, participants had the opportunity to practice, which was followed by the second test.

Following the second test, participants received failure feedback again, informing them that, "You correctly detected the emotions of 4 (out of 10) people whose photos you viewed. This means that your social perception skills are poor." Participants were led to believe that there would be another practice opportunity and a third social perception test, providing a cover to measure their current and anticipated emotions once more (Posttest 2 emotions). Afterward, they were thoroughly debriefed.

\section{Results}

\section{Overall Emotions}

To assess the relations between failure feedback, emotions, and practice, we reduced the five emotions measured by the BES into a single measure of negative emotion by computing the mean of 
participants' responses (reverse-coding positive emotion items), such that greater scores indicate relatively more negative emotions. All emotion measures (Baseline, Posttest 1, and Posttest 2 for both current and anticipated emotions) were extremely reliable, all $\alpha \mathrm{s}>$ .87. Given the strong reliability of the overall measure, all analyses were conducted on this single measure (see also, Phillips \& Silvia, 2005, for evidence that self-discrepancies influence both positive and negative emotions; see, Higgins, Shah, \& Friedman, 1997, for evidence that self-discrepancy effects are not moderated by emotion valence). However, for interested readers, descriptive statistics for all emotion measures, including the specific discrete emotions, are presented in Tables 1 and 2 .

Consistent with past research showing that self-discrepancies elicit relatively greater negative emotions (e.g., Phillips \& Silvia, 2005), participants' current emotions were more negative at Posttest 1 (after the first failure feedback) than at Baseline (at the beginning of the study), $t(203)=7.75, p<.001, d=.55$. Similarly, emotions were affected by the second failure feedback, with participants feeling more negative at Posttest 2 than Posttest $1, t(203)=2.93, p=.004, d=.21$. Thus, the (false) negative feedback was viewed as credible and had the intended impact of making participants' emotions more negative.

\section{Current and Anticipated Emotions as Predictors of Practice}

A path analysis was used to test the relations among current emotions, anticipated emotions, and practice (see Figure 1). All predictors were centered. In addition to the paths that represent the hypotheses, we included paths between repeated measurements (e.g., current emotions at Baseline and Posttest 1) to control for within-subject stability in responses. The number of minutes and of problems practiced were highly correlated $(r=.70, p<.001)$ and so we standardized and then summed the two to form a measure of practice effort (i.e., self-regulation), with greater scores indicating more practice effort.

First, we tested whether participants' current (discrepancybased) emotions immediately before the practice opportunity predicted their practice effort. Recall that classic theories of selfregulation predict that discrepancy-based negative emotions initiate self-regulation. However, contrary to predictions, participants

Table 1

Current Emotions by Measurement Time and Emotion Measure

\begin{tabular}{|c|c|c|c|c|c|c|}
\hline \multirow[b]{2}{*}{ Measurement } & \multicolumn{2}{|c|}{ Baseline } & \multicolumn{2}{|c|}{ Posttest 1} & \multicolumn{2}{|c|}{ Posttest 2} \\
\hline & $M$ & $S D$ & $M$ & $S D$ & $M$ & $S D$ \\
\hline Overall emotions ${ }^{\mathrm{a}}$ & 2.42 & 0.73 & 2.84 & 0.87 & 2.95 & 0.97 \\
\hline Negative emotions & 2.03 & 0.78 & 2.22 & 0.99 & 2.32 & 1.12 \\
\hline Positive emotions (happiness) & 4.02 & 1.19 & 2.67 & 1.41 & 2.53 & 1.40 \\
\hline Anger & 2.07 & 1.02 & 2.54 & 1.17 & 2.89 & 1.34 \\
\hline Sadness & 1.76 & 0.94 & 1.74 & 0.93 & 1.78 & 1.08 \\
\hline Self-disgust & 1.45 & 0.78 & 1.92 & 1.06 & 1.94 & 1.15 \\
\hline Fear & 2.84 & 1.37 & 2.69 & 1.45 & 2.68 & 1.59 \\
\hline
\end{tabular}

${ }^{a}$ Greater scores indicate more negative emotions. "Overall emotions" is the mean of all emotion items, with positive items recoded. ${ }^{\mathrm{b}}$ Greater scores indicate more positive emotion. This was the only positive emotion measured.
Table 2

Anticipated Emotions by Measurement Time and Emotion Measure

\begin{tabular}{|c|c|c|c|c|c|c|}
\hline \multirow[b]{2}{*}{ Measurement } & \multicolumn{2}{|c|}{ Baseline } & \multicolumn{2}{|c|}{ Posttest 1} & \multicolumn{2}{|c|}{ Posttest 2} \\
\hline & $M$ & $S D$ & $M$ & $S D$ & $M$ & $S D$ \\
\hline Overall emotions ${ }^{\mathrm{a}}$ & 3.34 & 0.99 & 3.20 & 1.03 & 3.31 & 1.18 \\
\hline Negative emotions & 2.66 & 1.22 & 2.53 & 1.25 & 2.66 & 1.40 \\
\hline Positive emotions (happiness) ${ }^{\mathrm{b}}$ & 1.91 & 1.05 & 2.09 & 1.28 & 2.07 & 1.33 \\
\hline Anger & 3.01 & 1.36 & 2.96 & 1.36 & 3.33 & 1.58 \\
\hline Sadness & 2.19 & 1.21 & 2.02 & 1.20 & 2.10 & 1.40 \\
\hline Self-disgust & 2.44 & 1.37 & 2.22 & 1.36 & 2.32 & 1.49 \\
\hline Fear & 2.98 & 1.52 & 2.91 & 1.65 & 2.90 & 1.76 \\
\hline
\end{tabular}

a Greater scores indicate more negative emotions. "Overall emotions" is the mean of all emotion items, with positive items recoded. ${ }^{\mathrm{b}}$ Greater scores indicate more positive emotion. This was the only positive emotion measured.

did not practice more when their emotions were more negative. In fact, there was a nonsignificant trend for participants to practice more when their emotions were more positive, $\beta=-.23, t(201)=$ $1.66, p=.10$.

Although current emotions did not predict practice, it was hypothesized that anticipated emotions would predict practice, such that participants would exert greater effort if they expected to feel more negative if they failed. Indeed, anticipated emotions at Posttest 1 significantly predicted practice effort, $\beta=.29, t(201)=$ $-2.07, p=.04$, such that participants practiced more as their expected feelings following failure became more negative.

\section{Learning: The Temporal Relation Between Current and Anticipated Emotions}

Affective forecasting research has shown that people are inaccurate when predicting how certain events will make them feel (e.g., Wilson \& Gilbert, 2003). Specifically, they overestimate the intensity and duration of their emotions. Thus, participants in this study might be expected to be inaccurate at predicting how they would feel following failure on the first test (the outcome all participants experienced). However, participants' anticipated feelings if they failed the first test predicted how they actually felt after learning they had failed the test, $\beta=.47, t(201)=8.69, p<.001$. Therefore, there was some accuracy in participants' predictions about how they would feel. However, a paired $t$ test revealed the overestimation bias typically observed in affective forecasting research, such that Baseline anticipated emotions after failure were significantly more negative $(M=3.34, S D=.99)$ than actual Posttest 1 emotions after failure $(M=2.84, S D=.87), t(203)=$ 8.46, $p<.001, d=.59$.

Important to the learning account of self-regulation, participants' actual emotions following the self-discrepancy should contribute to subsequent predictions of how they will feel when facing future self-discrepancies. As shown in Figure 1, current emotions at Posttest 1 (after failing the first test) did predict anticipated emotions at Posttest 1 (how participants expected to feel if they failed a second test), $\beta=.70, t(201)=18.22, p<.001$. Current emotions at Posttest 2 (after failing the second test) also predicted anticipated emotions at Posttest 2 (how participants expected to 


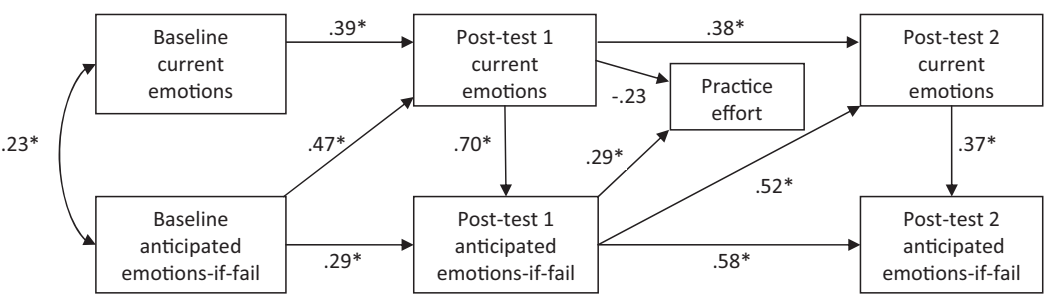

Figure 1. Path model with values representing standardized betas. ${ }^{*} p<.05$.

feel if they failed a third test), $\beta=.37, t(201)=6.77, p<.001$. In other words, the emotions participants felt after failing predicted the emotions they expected to feel if they were to fail again. This supports the assertion that discrepancy-based emotions guide subsequent anticipated emotions.

The second aspect of the learning account is that because people attend to their discrepancy-based emotions as useful information, the accuracy of their anticipated emotions should increase over time. In other words, participants' predictions about how they will feel after failure should become more accurate after repeated experiences of failure. First, we observed that anticipated emotions at Posttest 1 predicted current emotions at Posttest 2, $\beta=.52$, $t(201)=7.33, p<.001$, indicating that participants could predict how they would feel after a second failure. However, as with anticipated emotions at Baseline, participants' predictions $(M=$ $3.20, S D=1.03$ ) significantly overestimated the actual negativity $(M=2.95, S D=.97)$ of their feelings after a second failure, $t(203)=6.40, p<.001, d=.46$. On the other hand, the size of the discrepancy between anticipated and actual emotions $(M=$ $.50, S D=.85$ ) following the first failure was significantly greater than the difference between anticipated and actual emotions $(M=$ $.25, S D=.56)$ following the second failure, $t(203)=4.08, p<$ $.001, d=.29$, supporting the hypothesis that affective forecasts improve with experience. In other words, these data suggest that participants learned from their experiences and became more accurate at predicting future emotions. This is important given that these predictions (i.e., anticipated emotions) guide self-regulation.

\section{Model Fit}

The fit of the path model was significant using both the chisquare statistic, $\chi^{2}(10)=14.99, p=.13(p>.05$ indicates good fit; Kline, 2005) and the root mean square error of approximation, RMSEA $=.05$ (RMSEA $\leq .05$ indicates good fit; Kline, 2005).

\section{Discussion}

Contrary to classic self-regulation theories (e.g., Carver \& Scheier, 1999; Duval \& Wicklund, 1972), negative discrepancybased emotions did not predict self-regulation. In fact, there was a nonsignificant trend for participants to self-regulate more when their emotions were more positive following a self-discrepancy. At the same time, however, self-regulation was predicted by how people expected to feel given potential future outcomes. Specifically, participants practiced more when they expected to feel worse if they failed a test of an important ability. These data suggest that people are more attentive to how they think they will feel than how they currently feel when engaging in goal-directed behavior. How- ever, current feelings following a self-discrepancy predicted subsequent anticipated feelings, suggesting that people learn from their affective experiences, such that they inform their anticipated feelings, which do guide behavior. Indeed, the finding that participants' anticipated feelings became more accurate with experience (i.e., their overestimation bias was smaller the second time they predicted their emotions) is consistent with people attending to and using their current feelings to inform future judgments.

These results suggest that, in the context of goal-directed behavior, current emotions do not guide immediate actions but instead serve a different function. That is, they provide experience that is used to inform judgments (anticipated emotions) that do guide behavior, consistent with the perspective that emotions are not immediate causes of behavior (Baumeister et al., 2007). It seems likely that emotions have a distant effect on behavior by guiding attention (e.g., Fredrickson \& Branigan, 2005), directing cognitive processes (e.g., Forgas, 2001), and restructuring one's goal priorities (e.g., Simon, 1967), each of which, in turn, may have an immediate effect on behavior.

Although the results of this study contribute to our understanding of the relation between emotions and goal-directed behavior, there are a number of questions that remain about the exact nature of anticipated emotions in self-regulation. For example, future research should explore if the type of anticipated emotion influences self-regulation. We only explored how participants expected to feel if they failed because all participants actually received failure feedback, enabling us to examine the accuracy of these predictions and their improvement following experience. It would be interesting to explore if self-regulation is also guided by how people anticipate feeling if they succeed, although such a relation could be moderated by individual differences (e.g., prevention and promotion focus; Higgins, 1997) and the situation (e.g., whether the person recently experienced a failure or success). Future research should also compare the relation between discrepancybased emotions and other emotions on self-regulation.

A limitation of the current study is that we did not include a no-discrepancy control condition to determine whether nondiscrepancy emotions predict self-regulation. However, neither Baseline current emotions, $\beta=-.06, t(201)=.81, p=.42$, nor Baseline anticipated emotions, $\beta=.07, t(201)=.89, p=.38$, were significant predictors of practice when controlling for each other, suggesting that the context of a self-discrepancy was necessary for the relation between anticipated emotions and selfregulation observed in this study. Finally, it would be beneficial to replicate the results of the current study using physiological and behavioral indexes of emotion to circumvent the potential for 
demand characteristics in self-reported emotion, which is a limitation of the current study.

Additional research also should explore different types of goaldirected behavior, such as different goal domains and situations in which people have no prior feedback about their ability. Moreover, there is considerable research on how attributes of the selfdiscrepancy influence emotions (e.g., Boldero \& Francis, 2000; Phillips \& Silvia, 2005), but the relation between these attributes, emotions, and actual self-regulatory behavior has not been studied. There may also be exceptions in which current emotions do predict self-regulation, such as when attention to affect is primed or among people who chronically attend to their emotions (e.g., Brown \& McConnell, 2009).

One final area where the current findings may shed light is our understanding of how people render predictions of their future emotional experiences. Indeed, work from the affective forecasting literature suggests that people are relatively poor at anticipating their affective reactions to yet-to-be-experienced events, and that they are especially prone to overestimate both the intensity and duration of their emotional responses (Dunn \& Laham, 2006; Wilson \& Gilbert, 2003). The current work shows that although people may not be completely accurate in their predictions of future emotions, they are reliable in their ability to anticipate their affective reactions. More important, the design of the current work allowed an examination of how these predictions improve with time, and indeed, people's accuracy (i.e., the discrepancy between their predicted and actual emotions) improved. Although these findings are admittedly preliminary in nature, they indicate that learning about one's emotional reactions occurs, and that affective forecasting errors may diminish with experience and time. Future work should examine how learning may moderate affective forecasting errors.

\section{Conclusions}

Considerable research has established that self-discrepancies evoke negative emotions, yet the function and consequences of these emotions have been relatively ignored. The current work reveals that these discrepancy-based emotions may serve an informational role in self-regulation, a function that differs from classic conceptualizations of negative emotions in self-regulation. Rather than directing immediate goal-directed behavior, one's emotions can provide a foundation from which future expectations-which do guide behavior - are based. Regarding the function of emotions more generally, these findings are consistent with the perspective that emotions are not a direct cause of behavior, but rather may affect it indirectly, such as by creating expectations, prioritizing goals, and guiding attention.

\section{References}

Baumeister, R. F., Vohs, K. D., DeWall, C. N., \& Zhang, L. (2007). How emotion shapes behavior: Feedback, anticipation, and reflection, rather than direct causation. Personality and Social Psychology Review, 11, 167-203.
Boldero, J., \& Francis, J. (2000). The relation between self-discrepancies and emotion: The moderating roles of self-guide importance, location relevance, and social self-domain centrality. Journal of Personality and Social Psychology, 78, 38-52.

Brown, C. M., \& McConnell, A. R. (2009). Effort or escape: Self-concept structure determines self-regulatory behavior. Self and Identity, 8, 365377.

Carver, C. S., \& Scheier, M. F. (1999). Themes and issues in the selfregulation of behavior. In R. S. Wyer, Jr. (Ed.), Perspectives on behavioral self-regulation: Advances in social cognition (Vol. 12, pp. 1-105). Mahwah, NJ: Erlbaum.

Cooper, J., \& Fazio, R. H. (1984). A new look at dissonance theory. In L. Berkowitz (Ed.), Advances in experimental social psychology (Vol. 17, pp. 229-266). Orlando, FL: Academic Press.

Cosmides, L., \& Tooby, J. (2000). Evolutionary psychology and the emotions. In M. Lewis \& J. M. Haviland (Eds.), Handbook of emotions (2nd ed., pp. 91-115). New York, NY: Guilford Press.

Damasio, A. R. (1994). Descartes' error: Emotion, reason, and the human brain. New York, NY: Putnam.

Dunn, E. W., \& Laham, S. M. (2006). Affective forecasting: A user's guide to emotional time travel. In J. P. Forgas (Ed.), Affect in social thinking and behavior (pp. 177-193). New York, NY: Psychology Press.

Duval, S., \& Wicklund, R. A. (1972). A theory of objective self-awareness. New York, NY: Academic Press.

Forgas, J. P. (Ed.). (2001). Handbook of affect and social cognition Mahwah, NJ: Erlbaum.

Fredrickson, B. L., \& Branigan, C. (2005). Positive emotions broaden the scope of attention and thought-action repertoires. Cognition \& Emotion, 19, 313-332.

Frijda, N. H. (2000). The psychologists' point of view. In M. Lewis \& J. M. Haviland (Eds.), Handbook of emotions (2nd ed., pp. 59-74). New York, NY: Guilford Press.

Higgins, E. T. (1987). Self-discrepancy: A theory relating self and affect Psychological Review, 94, 319-340.

Higgins, E. T. (1997). Beyond pleasure and pain. American Psychologist, 52, 1280-1300.

Higgins, E. T., Shah, J., \& Friedman, R. (1997). Emotional responses to goal attainment: Strength of regulatory focus as moderator. Journal of Personality and Social Psychology, 72, 515-525.

Kline, R. B. (2005). Principles and practices in structural equation modeling (2nd ed.). New York, NY: Guilford Press.

Minear, M., \& Park, D. C. (2004). A lifespan database of adult facial stimuli. Behavior Research Methods, Instruments, \& Computers, 36, 630-633.

Phillips, A. G., \& Silvia, P. J. (2005). Self-awareness and the emotional consequences of self-discrepancies. Personality and Social Psychology Bulletin, 31, 703-713.

Power, M. J. (2006). The structure of emotion: An empirical comparison of six models. Cognition \& Emotion, 20, 694-713.

Simon, H. A. (1967). Motivational and emotional controls of cognition. Psychological Review, 74, 29-39.

Wilson, T. D., \& Gilbert, D. T. (2003). Affective forecasting. In M. P. Zanna (Ed.), Advances in experimental social psychology (Vol. 35, pp. 345-411). San Diego, CA: Academic Press.

Received June 8, 2010

Revision received September 23, 2010 Accepted September 24, 2010 